\title{
LECTURA EN DERECHO: SUGERENCIAS PARA GUIAR AL ESTUDIANTE EN SU PROCESO FORMATIVO Y EN LA APROPIACIÓN DEL CONOCIMIENTO
}

\author{
Jesus Morales * \\ https://orcid.org/0000-0001-8533-3442
}

RECIBIDO: Octubre 2020 / ACEPTADO: Enero 2021 / PUBLICADO: Mayo 2021

Como citar: Morales, Jesus (2021). Lectura en derecho: sugerencias para guiar al estudiante en su proceso formativo y en la apropiación del conocimiento. Telos: revista de Estudios Interdisciplinarios en Ciencias Sociales, 23 (2), Venezuela. (Pp.227-246).

DOI: www.doi.org/10.36390/telos232.03

\section{RESUMEN}

Los ingresantes a la universidad se ven enfrentados a una cultura académica diferente de la que provienen. Para integrarse a ella, han de asumir nuevos roles como lectores (además de otros cambios sociales y cognitivos). Las dificultades lectoras con las que se encuentran provienen de su inexperiencia en las formas de lectura que la universidad exige. Partiendo de las dificultades habituales con las que los estudiantes que ingresan a la Universidad se encuentran al abordar textos jurídicos, se busca describirlas estrategias de lectura que caracterizan a los expertos en este campo para así, en un futuro, contar con herramientas más eficaces de enseñanza Se hace notar la importancia de que los alumnos sean estimulados a leer las sentencias con un propósito definido, pongan el fallo en contexto, conozcan las características estructurales de los textos, los aborden de manera flexible y realicen una evaluación crítica del fallo. En este artículo presentamos los principales aspectos de la reflexión hecha en torno a la lectura en la clase de ciencias y que ayudaron a introducir cambios en la práctica docente y proponer estrategias para realizar lectura crítica en el aula universitaria. Los autores citados para apoyar esta investigación se encuentran: Borja (2007), Knorr (2010), Moretó (2014) y Weston (2006).

Palabras clave: comprensión lectora; estrategias de lectura; textos jurídicos; autonomía; enseñanza; lectura crítica.

* Universidad de Los Andes, Venezuela. Correo Electrónico: lectoescrituraula@gmail.com 
Lectura en derecho: sugerencias para guiar al estudiante en su proceso formativo y en la apropiación del conocimiento

\title{
Reading in law: suggestions to guide the student in his formative process and in the appropriation of knowledge
}

\begin{abstract}
Freshmen meet a different academic culture compared with their previous experiences and in order to get immerse in it, they have to play new roles as readers (besides other cognitive and social changes). From the usual difficulties the first-year law students find when they read legal texts, we search to describe and compare the strategies of reading that characterized experts, to have in future tools for teaching in a more effective way. It is noted the importance of encouraged students to read sentences with a specific purpose, to put the decision into context, to be aware of the structural features of the texts, to address it in a flexible way and to make a critical assessment of the opinion. This article we present here the main aspects of the reflection concerning reading in science class which helped to bring about changes in our teaching practice and propose strategies for achieving critical reading at the university level. The authors cited to support this research are found: Borja (2007), Knorr (2010), Moretó (2014) y Weston (2006).
\end{abstract}

Keywords: reading comprehension; strategies of reading; legal texts; autonomy; teaching; critical reading.

\section{Introducción}

Leer en el campo de las ciencias jurídicas representa una de las tareas más complejas a las que se enfrenta el estudiante de nuevo ingreso. Esto se debe en gran medida a las debilidades que los lectores novatos tienen al acercarse al conocimiento científico y cuya razón de ser se encuentra determinada por la ausencia de prácticas lectoras pertinentes y significativas que procuren el desarrollo de habilidades del pensamiento superior como la crítica, la reflexión, el análisis y el razonamiento por mencionar algunas.

En este sentido, las prácticas de lectura en la universidad se han asumido en la actualidad como una poderosa herramienta al servicio de los procesos de enseñanza y aprendizaje de las disciplinas. Esto desde las exigencias formativas propias del campo jurídico no varía, pues se trata de acercar de modo activo a las fuentes de conocimiento (sentencias, jurisprudencias, contratos, doctrina, entre otras) a las que debe apelar el estudiante para construir de manera autónoma y responsable nuevas ideas y alternativas que aporten a la resolución de conflictos.

En Weston (2006), la lectura en derecho además de entenderse como un proceso inherente a la educación del futuro profesional, también se relaciona con la elaboración minuciosa de argumentos lógicos y válidos, recursos que dentro de toda querella judicial le permiten al jurista, juez o defensor sustentar su posición con alegatos convincentes, con pruebas pertinentes y razones rigurosamente pensadas que logren adherir a la audiencia y a las partes en torno a una posición.

Para Gordillo (2000), el lector de las disciplinas socio-jurídicas debe asumir como premisa que "los hechos son lo más importante en derecho, debido a que si nos equivocamos en los hechos todo lo demás no sirve" (p.135). Para el autor, el conocimiento de los hechos contribuye con la deducción del carácter justo o injusto de la solución aplicada, condición que 
para el lector crítico representa la manera de determinar los excesos, arbitrariedades y mala praxis en la que pudo haber incurrido el administrador de justicia.

Por tal motivo y considerando la necesidad de ofrecer algunas aproximaciones sobre la lectura en el campo del derecho, se propone el siguiente ensayo; el cual pretende, además de discutir las bondades de la lectura para el que se forma en la universidad en un área tan compleja como el derecho, también deja ver que, tanto para el que aprende como para quien enseña, las estrategias que se pudieran asumir con el propósito de no perderse en la densa y abundante información con la que debe interactuar el abogado en formación.

\section{Lectura de textos jurídicos}

Formar para la vida y la participación en el mundo social, además de definirse como ideales inherentes al desarrollo humano, también se han consolidado como premisas estrechamente relacionadas con la construcción de espacios democráticos en los que halle espacio la justicia, el bien común y la seguridad jurídica. Al respecto, la lectura académica deja ver que el fortalecimiento de estos valores se encuentra soportado en procesos educativos pertinentes, en los que el sujeto sea sensibilizado para ejercer con responsabilidad su ciudadanía, condición que según las tendencias de la formación crítica le viene dada mediante la interacción significativa con la información y el conocimiento.

Lo anterior indica que, la lectura como proceso sociocultural entraña bondades como el ingreso competente al mundo letrado así como a la comprensión de las prácticas sociales que hacen parte de las relaciones humanas, entre las que se destaca la administración efectiva de justicia como el bien preciado del que depende el equilibrio institucional y el bienestar social, por mencionar algunos. De este modo, pensar en la lectura como herramienta al servicio del desarrollo democrático de cualquier sistema, implica verla en sentido amplio como el medio para reducir las desigualdades, pero además, como un mecanismo capaz de garantizar elevados niveles de responsabilidad y participación en los asuntos públicos que nos atañen a todos.

Durante los últimos años, estos cometidos han reportado medular importancia para quien se forma en áreas específicas como las ciencias jurídicas, profesional en sobre el que recae la construcción de procesos decisorios objetivos y ajustados a derecho, también se le confía el rol de quien haciendo uso de su sentido crítico y de la experticia, debe ser capaz de responder a las necesidades sociales que recurrentemente emergen como resultado de la dinámica social. Lograr estas pretensiones sin lugar a duda representa un reto para quien se forma en el campo jurídico, le exige el uso de competencias lectoras, que impulsen la valoración del mundo de modo ecuánime, proceso en el cual es indispensable la interacción con el conocimiento reflexivamente, valiéndose de habilidades cognitivas como: la integración de información, definición de posiciones objetivas y la elaboración de soluciones, como máximas en función de las cuales actuar en apego a la legalidad y a la justicia, como condiciones imprescindibles para sustanciar y legitimar sus decisiones (Alvarado, 2014; Morales, 2018; Moretó, 2014).

Atienza (2005), haciendo referencia a las formas particulares como se enseña el conocimiento científico jurídico, expone las dificultades que enfrenta el estudioso del derecho, estas por lo general, giran en torno al aprendizaje estricto y literal de las normas, al que se le ha sumado un explícito apego a procesos de memorización de principios interpretativos que, 
Lectura en derecho: sugerencias para guiar al estudiante en su proceso formativo y en la apropiación del conocimiento

por no estar contextualizados y explicados a la luz del derecho vigente, se han convertido en lastres que imposibilitan el acercamiento crítico, reflexivo y analítico al contenido subyacente en los sistemas normativos y al verdadero espíritu que motivó al legislador en la producción de las normas, sentencias y jurisprudencias.

En atención a lo dicho, las perspectivas de la educación en el siglo XXI han sufrido modificaciones significativas, motivadas fundamentalmente por la necesidad de generar procesos y experiencias de enseñanza-aprendizaje en los que al sujeto se le entiende como agente activo con el potencial para asumir con responsabilidad el rol protagónico desde el cual operar sobre la realidad, apropiarse de la información y construir de manera autónoma su propia visión de lo sucede en el mundo. Leer en Derecho es entonces, un proceso en el que participan operaciones mentales como el análisis y la interpretación, mediante las cuales el lector busca desentrañar tanto el significado de las normas jurídicas como su contenido e implicaciones sociales, de las cuales dependerá que el colectivo y el sistema de justicia logren construir soluciones en las que impere el derecho y las máximas sobre las que este se soporta para garantizar el orden social y el equilibrio en los vínculos humanos.

De este modo, leer se entiende como un proceso crítico con implicaciones significativas en la construcción de conocimiento a partir de la integración de las diversas y múltiples miradas existentes sobre un fenómeno o hecho social, a partir del cual proponer posiciones y enfoques que integrados permiten ampliar la visión de quien se acerca al saber con sentido crítico y reflexivo. Estas bondades son igualmente compartidas por Knorr (2010),

qa para quien la lectura crítica propicia que el sujeto "se introduzca en el tema 0 problema, identifique características, los antecedentes 0 historia, analice las partes 0 componentes y, como resultado de ello elabore conclusiones" (p.16). Esto posiciona a la lectura en Derecho como una exigencia capaz de atender varios factores, por un lado, la búsqueda de la verdad a través de una actitud valorativa, acuciosa y reflexiva, con el potencial de orientar en el proceso de definir posibles alternativas de solución a los problemas sociales $y$, por el otro, asumir con responsabilidad su rol como administrador de justicia, el cual debe apegarse a principios tales como: equidad, igualdad, bien común y justicia; de la unión de estos dos aspectos se espera el proceder idóneo ante las complejas situaciones jurídicas.

A lo anterior se agrega que, la dinámica social ha propuesto nuevas exigencias formativas, en las que se han incluido conceptos valiosos y actividades relacionadas con el desarrollo del pensamiento, el uso de la criticidad y de la reflexión como medios para ingresar a la cada vez más abundante y compleja información generada desde las comunidades científicas; de allí que, una de las maneras propicias de enfrentar este reto, se haya traducido en "la necesidad de guiar procesos de aprendizaje en los que el estudiante logre seleccionar, interpretar, asimilar, procesar y finalmente expresar con claridad sus propios conocimientos" (Ulloa, Crispín y Béjar, 2014, p.1).

Eso significa que, la formación crítica del ciudadano de este siglo, se encuentra más que nunca determinada por la necesidad de integrarse en el escenario ciudadano, social y académico como ámbitos que por la relevancia de sus prácticas constituyen variables vinculadas con el desarrollo de cualquier país; de allí que, las acciones institucionales y pedagógicas hayan incluido en sus planes prácticas de lectura crítica como una herramienta que, además de permitir el funcionamiento coherente de la vida democrática también cuenta con el potencial para desarrollar elevados niveles de participación en la vida social mediante 
los cuales comprometer su actuar con la transformación efectiva de sus propias condiciones de convivencia.

Lo dicho además de obedecer a los cometidos de la lectura crítica, también indica que del estudiante de nuevo ingreso se espera entre otras cosas, que proceda con actitud analítica y reflexivamente frente al conocimiento; lo que implica valorar la información desde un enfoque interdisciplinario del cual depende su aproximación a las diversas miradas sobre su objeto de estudio. Para Hawes (2003), la lectura acuciosa de textos académicos trae beneficios significativos para el sujeto, entre las que se mencionan "permite la construcción de su propia voz, la participación pertinente en la discusión de temas del acontecer social y proceder de manera autónoma y responsable frente a las necesidades de su entorno" (p.41).

En atención a lo anterior, la enseñanza de la lectura en el campo de las ciencias jurídicas no escapa de ser semejante a la que se desarrolla en otras disciplinas, pues se tiene como propósito acercar al estudiante a las diversas voces que subyacen en los géneros académicos y de las que depende el desempeño coherente del abogado en su proceso formativo y en el campo profesional al que se integrará. Parafraseando a Guerra (2006) leer en un área compleja como las ciencias jurídicas requieren de procesos críticos a partir de los cuales analizar la información que contienen los textos de la disciplina. En palabras del mismo autor, esto es importante por varias razones, entre las que se pueden destacar:

1. Quien tiene a su cargo la toma de decisiones dentro de los procesos judiciales, debe desentrañar ideas e identificar planteamientos subyacentes en con el propósito de evitar errores que le pudieran llevar a posiciones subjetivas 0 alejadas de los principios que deben guiar el proceso.

2. La construcción de alegatos efectivos depende de revisiones reflexivas y críticas de otras experiencias que, mostradas en sentencias y jurisprudencias, permiten que el juez, el abogado y los administradores de justicia logren sustanciar sus posiciones.

3. La lectura crítica de los géneros jurídicos representa un medio indispensable para la elaboración de planteamientos coherentes, pero además, como un instrumento para enfrentar $\mathrm{y}$ detectar argumentos falaces o defectuosos.

En palabras de Moretó (2014), leer en el ámbito jurídico requiere de la puesta en funcionamiento de "objetivos y propósitos de lectura específicos como el cuestionar, buscar información relevante, hacer inferencias, sintetizar y monitorear la construcción de significado" (p.100). Lograr estos cometidos implica, ser capaz de rastrear datos importantes dentro de los géneros jurídicos, en el caso de una sentencia: nombres de los apartados, identificación de la fecha en que fue emitida, tribunal y juez encargado de la causa. Otras posiciones afirman que, el lector en su proceder activo debe identificar otros aspectos importantes tales como:

1. Identificar los sujetos de derecho involucrados, es decir, el sujeto pasivo a quien se dirige el accionar de alguna exigencia por la vulneración de un derecho y, el sujeto activo, quien en el ejercicio de su posición acude ante una instancia judicial para que se le resarza por el daño causado.

2. Es fundamental que el lector determine las motivaciones que tuvo el administrador de justicia o los considerandos expuestos en una sentencia.

En el caso de otros géneros jurídicos como las leyes y otras normas, es imprescindible que el lector ahonde sobre otros aspectos necesarios para comprender las intencionalidades subyacentes, es decir, revisar el contexto sociopolítico en el que fue sancionada o creada la 
Lectura en derecho: sugerencias para guiar al estudiante en su proceso formativo y en la apropiación del conocimiento

misma. Lo anterior, demanda la recogida de datos que una vez registrados definan tanto el régimen de sanciones como el de infracciones, es decir, el tipo de conductas potencialmente susceptibles de sanción, esto refiere a la atribución de responsabilidad (civil, penal y administrativa) que se puede derivar de la aplicación de la ley.

Para Anchondo (2016) y Weston (2006), la lectura en este campo debe guiar al estudiante a la identificación y clasificación de los documentos, partiendo de operaciones como la caracterización, es decir, la tipificación de aspectos tanto de forma como de fondo que se integran en los escritos manejados en el campo jurídico. En este sentido, la identificación del documento permite entre otras cosas lograr ubicarlo dentro de una fuente jurídica específica, lo cual implica definir si obedece a una sentencia, una jurisprudencia, una ley, un reglamento 0 un cuerpo doctrinal en el que se desarrollan elementos teóricos, conceptuales e históricos que el estudiante debe manejar para comprender la perspectiva desde la cual se está abordando su objeto de estudio.

Al respecto, Moretó (2014), propone que atender a "las características de un texto jurídico en su forma o estructura facilita procesos de comprensión más fluido" (p.100). Siguiendo al autor, la organización del texto favorece que el lector logre entre otras cosas y, en el caso específico de un fallo judicial la identificación de los siguientes aspectos: la sinopsis, luego definir las relación de hechos, exposición de antecedentes, los considerandos, la disposición y los elementos doctrinales sobre los que sustenta la decisión.

Otras visiones, proponen que leer en derecho parte de un proceso académico consistente en situar el documento o género dentro de un área en específico según su naturaleza, elementos contenidos y asunto tratado o controversia expuesta. Esto implícitamente apunta a la ubicación dentro del ámbito público o privado, penal, administrativo, civil, mercantil o laboral, o en caso excepcional conviven elementos provenientes de varias materias que, el estudiante debe lograr dilucidar a través de la comprensión de las motivaciones, sujetos involucrados y materias tratadas.

Para alcanzar tales cometidos, la lectura académica ha dejado ver la necesidad de apelar a la revisión sistemática de otras fuentes que le permitan completar aspectos vacíos, condición que exige del estudiante un elevado compromiso formativo consistente en integrar los conocimientos provenientes de jurisprudencias, comentarios realizados en doctrina sobre el caso, así como en otros materiales cuya utilidad hace posible el ingreso al conocimiento de áreas específicas dentro de su disciplina.

Según Pettoruti (2005), leer en derecho requiere del uso del pensamiento para "relacionar conceptos y vincular conocimientos, pero además ser capaces de conocer e interpretar normas y hechos en relación con la realidad social" (p.102). Esto exige dilucidar el ámbito de aplicación de la fuente jurídica revisada, es decir, si la misma tiene alcance nacional o internacional, o si su operatividad se limita exclusivamente a un espacio geográfico determinado dentro de un país.

Al respecto, Guerra (2006), propone que la lectura de textos jurídicos está relacionada con la formación de lectores críticos, capaces de operar sobre la información de la siguiente manera: leer requiere de habilidades para conjeturar y objetar las afirmaciones de terceros con una actitud responsable y honesta; como resultado de lo anterior, el lector debe resistir los efectos de la manipulación, cuya intención es guiar a la construcción de ideas erradas; la elaboración de interrogantes le permite al lector reflexionar sobre lo que sabe y lo que requiere 
buscar en otros textos para completar ideas; el lector crítico debe fundar sus juicios y valoraciones en evidencias; establecer conexiones entre temas, ideas y posiciones teóricas, facilita mayores posibilidades de ingresar a nuevas alternativas que permitan resolver una controversia.

Por su parte, estudiosos de las tramas argumentativas que caracterizan a los textos jurídicos proponen que, el lector de este campo debe desarrollar habilidades cognitivas que le cooperen con la identificación de aspectos tales como: el punto de vista o la idea que el autor pretende defender y de la que espera sea compartida por su audiencia; identificar los argumentos (citas, ejemplos, estadísticas y recursos analógicos a los que apela para convencer o persuadir, por el hecho de representar pruebas o razonamientos que le aportan rigurosidad y validez a sus afirmaciones.

Dolz y Pasquier (1997), al respecto proponen que comprender el discurso argumentativo que predomina en el derecho, requiere de una lectura capaz "anticipar globalmente la posición del destinatario, justificar y desarrollar su punto de vista a partir de sus argumentos, a los cuales debe atender con sentido crítico, definiendo con ello su acuerdo o desacuerdo" (p.10). Lo dicho desde la lectura crítica no es más que identificar las intenciones de las opiniones expresadas y las pretensiones de sus argumentos, en los cuales subyacen elementos ideológicos que buscan receptores que se adhieran a su postura.

Por ende, leer en este campo del saber debe enfocarse en guiar al lector hacia la identificación de estructuras básicas o componentes caracterizados por contener una tesis que pudiera estar acompañada de sub-tesis, cuya función junto a los argumentos no es otra que permear de la fuerza necesaria para persuadir a terceros de los que se espera su adhesión. Esta competencia argumentativa refiere a: la búsqueda de razones válidas y confiables y la articulación de premisas e ideas a partir de las cuales sustentar sus propias conclusiones y generalizaciones.

De este modo, leer en el campo jurídico exige la búsqueda de sentido y de coherencia en las ideas propuestas, pero además, las competencias para deducir relaciones causa-efecto cuya presencia es recurrente en las sentencias judiciales, la identificación de los sujetos pasivo y activo, así como las relaciones espacio-temporales que subyacen a la controversia. A esto agrega León (2008) que el lector en su proceder analítico debe acercarse a la identificación del problema central del caso en cuestión así como de los elementos secundarios que por su relevancia favorecen la reconstrucción de los hechos (sujetos involucrados indirectamente, condiciones, entre otros) los cuales sirven de insumos para orientar procesos decisorios.

Para otras perspectivas de la lectura en el derecho, esta actividad se encuentra vinculada con procesos interpretativos que le coadyuvan al lector con el ejercicio de competencias propias de su área como: descubrir, desentrañar y explicar el verdadero sentido y las intenciones que el legislador dejó implícitas en la norma. Lograr lo dicho requiere de la participación del sentido crítico para deducir aquellos elementos y disposiciones contradictorios o que no se encuentran explícitamente planteados en la norma.

Esto desde el punto de vista académico, le permite al lector apropiarse de ideas que sustancien su posición y, desde la praxis instrumental se entiende como una manera de persuadir a un tercero del que se espera responda a sus exigencias o propuestas. Para Pascual (1997) la lectura coopera con la identificación de secuencias discursivas con las que se debe familiarizar el abogado en formación, debido a que sobre este reposa el compromiso 
Lectura en derecho: sugerencias para guiar al estudiante en su proceso formativo y en la apropiación del conocimiento

de "narrar hechos, redefinir sucesos, tomar posición, se ubica desde una perspectiva determinada y desde allí reconstruye con fines que, en la mayoría de los casos es persuasivo" (p.2).

Se trata entonces de entender a la lectura como el proceso cognitivo y social que procura entre otras cosas, lograr que el estudiante se enfrente con eficacia a los escritos de su disciplina, afiliándose a prácticas tales como: integrar conocimiento, y desarrollar habilidades relacionadas con el aprendizaje autónomo que le permitan participar de manera pertinente en los actos comunicativos que hacen parte de la praxis del jurista. En apoyo a lo dicho Arenas (2009) propone que el lector universitario dentro de su vida académica debe operar responsablemente y con propósitos claramente definidos como fortalecer su "capacidad discursiva, lógica y analítica que le permita acceder a diversos tipos de textos en los que se requiera identificar, contrastar, abstraer, analogar, inferir, criticar, argumentar, contraargumentar, además de integrar los saberes aportados en otras áreas del conocimiento relacionadas" (p.2).

Hawez (2003), coincide con la propuesta anterior, al definir que la lectura crítica representa una herramienta indispensable de la que no solo depende la revisión profunda de ideas sino el desarrollo de habilidades vinculadas con "ser capaz de analizar, evaluar y sintetizar las relaciones existentes entre ideas, en las que a su vez debe identificar planteamientos centrales, argumentos en favor y en contra y, conclusiones" (p.42). Lo dicho refiere al compromiso que recae sobre el lector de materiales jurídicos, quien además de delimitar el propósito central del autor, debe poner en evidencia las múltiples intenciones subyacentes a partir de las cuales ser capaz de ofrecer explicaciones mediante las cuales argumente su propia posición frente a un tema determinado.

Para Knorr (2010), el acercamiento al conocimiento científico exige que, el lector universitario se ejercite en el manejo de los aspectos relevantes que giran en torno la explicación de un tema y las relaciones con aportes elaborados en otras disciplinas a las que debe apelar para definir otras miradas, que al ser integradas favorecen la comprensión de las problemáticas abordadas por la materia que se está estudiando.

La posición de Weston (2006), es oportuna para definir que, el lector de las disciplinas jurídicas debe interesarse por la búsqueda acuciosa de la verdad, actitud que refiere a procesos como "el cuestionamiento de sus propias creencias, proceso consistente en someter a prueba y dilucidar la solidez de sus propias opiniones" (p.14). Esta responsabilidad implica que, el estudiante asuma su aprendizaje por sí mismo, es decir, desarrolle la disposición objetiva para aproximarse a los problemas y cuestiones propias de su disciplina, con el propósito de lograr la elaboración de generalizaciones que apoyen su postura.

Para Gimeno (2000), el rol de quienes enseñan Derecho debe trascender la elaboración interpretaciones y análisis aislados y repetitivos en los que el estudiante no logra desarrollar competencias críticas como herramientas inherentes a la formación jurídica; de allí que su propuesta sea "acercar a quienes se inician en el campo a procesos en los que describan los posibles significados de los textos jurídicos en lugar de proponer uno, esto pudiera resolver los problemas generados por la vaguedad y la ambigüedad" (p.706).

Otras posiciones sobre la lectura de doctrina jurídica, manifiestan que es necesario revisar "los argumentos en los que se encuentra justificada una decisión, definiendo su validez y sentido lógico, con el propósito de no caer en interpretaciones vacías y falacias que alejen al 
lector de la construcción de planteamientos que le aporten soluciones razonables" (Suarez y Conde, 2009, p.21). Esta manera de operar con la información exige la revisión de las premisas sobre las que soporta el autor su posición, pero además, lograr establecer conexión entre premisas y las conclusiones; en virtud de ello, es posible determinar con certeza la validez de la posición propuesta por el autor.

Para Socorro y Cruceta (2007), la lectura crítica en derecho demanda de quien se forma en este campo disciplinar la "revisión de los enunciados o presupuestos en un argumento, pues los mismos pueden resultar claves a la hora de evaluar su fuerza" (p.113). Este modo crítico de proceder busca entre otras cosas, determinar los aspectos débiles y la veracidad de las afirmaciones, que por encontrase de manera encubiertas pudieran derivar en la elaboración de conclusiones falsas e imposibilitando la reconstrucción verdadera de planteamientos que apunten a esclarecer las verdaderas intenciones del autor.

En resumen, la lectura de documentos jurídicos (jurisprudencia, sentencias, contratos, entre otros) debe llevar al estudiante desarrollar competencias interpretativas, es decir, identificar el verdadero sentido en los textos, la tesis y los argumentos sobre los que se sustenta, elementos de los que depende la posterior reconstrucción del texto base; competencias argumentativas que orienten en determinar la organización de premisas, de razonamientos y afirmaciones a las que el autor apela para sustanciar su conclusión; y, la competencias propositiva, como herramienta inherente al estudioso de la ciencia jurídica, quien por ser administrador de justicia debe ser competente para elaborar hipótesis, delinear alternativas de solución a los conflictos sociales y predecir posibles escenarios que se pudieran presentar en su praxis.

\section{Estrategias para la lectura de textos jurídicos}

La lectura en Derecho requiere el manejo de estrategias relacionadas con el quehacer activo del lector, es decir, de la disposición reflexiva y del sentido crítico como procesos necesarios en la apropiación del conocimiento, en la detección de debilidades 0 falencias y en la búsqueda de la verdadera intencionalidad de las normas y demás géneros propios del campo jurídico. Al respecto, Knorr (2010), plantea que aprender y construir conocimiento representan procesos vinculados, con el potencial de facilitar la revisión profunda y la valoración significativa de ideas, planteamientos y posturas a partir de los cuales "integrar progresivamente temáticas y conceptos centrales que cooperen con la elaboración de propósitos centrales que subyacen en todos los textos académicos" (p.15). Por su parte, Gordillo (2000), refiriéndose a la interacción significativa con la información jurídica, propone la necesidad de disponer cognitivamente elevadas dosis de análisis, facilitándole al lectora la ubicación de "los puntos nodales o coyunturales, los ejes fundamentales de aquello que estamos leyendo" (p.116). Ello exige prestar especial atención a las condiciones históricas, económicas y sociales, como factores motivares de su promulgación y, cuyo contenido explicito le permiten al lector recoger los antecedentes y los puntos resolutivos, en función de los cuales dirimir una querella judicial.

Para Borja (2007), la lectura académica de los géneros jurídicos puede darse a partir del enfoque que tenga el lector, es decir, puede dedicarse a la revisión de los aspectos intratextuales que refieren a elementos sintácticos, pragmáticos y léxicos, mientras que la lectura del discurso legal se ocupa de la profundización en aquellos elementos extratextuales 
Lectura en derecho: sugerencias para guiar al estudiante en su proceso formativo y en la apropiación del conocimiento

que favorecen el trascender en la búsqueda de ideas subyacentes en los textos, tales como: los rasgos históricos de una norma, las condiciones culturales y las características sociales que dominaban para el momento de la elaboración de la norma, jurisprudencia o sentencia juridicial.

Para Bhatia (1997), la lectura significativa del conocimiento científico, requiere el manejo de competencias de comprensión específicas entre las que se mencionan:

1. Apropiarse los significados y usos que se le da a la terminología especializada; esto implica apelar a diccionarios jurídicos que aporten claridad sobre sus acepciones dentro de la disciplina.

2. Profundizar en la revisión de los códigos comunicativos de manejo frecuente dentro del área.

3. Identificar los contextos en los que se utilizan determinadas prácticas comunicativas y retóricas de las que depende la correcta resolución de conflictos y situaciones nuevas e inesperadas.

Por otra parte, leer en ciencias jurídicas se torna una actividad compleja no solo por el elevado uso de tecnicismos, sino por el extenso entramado teórico que se elabora desde las instituciones administradoras de justicia y sobre el cual se soportar el avance del conocimiento jurídico. A esto se agrega la construcción de un discurso saturado múltiples voces y de largas discusiones, antecedentes y abundancia de citas que el lector debe conocer, pero que, no debe representar un punto sobre el cual fijar su atención, por el contrario debe disponer el pensamiento para ubicar en el documento lo que se resuelve y los procedimientos seguidos por juez frente a una querella judicial (Gordillo, 2000; Guerra, 2006; León, 2008; Weston, 2006). Esto implica, la búsqueda de la situación de hecho a la que implícita o explícitamente hace referencia el fallo, dejando ver cuál es el problema que se trata y la decisión tomada por el tribunal; entender estos elementos significa aproximarse a "la descripción del conflicto, los argumentos de derecho argüidos, qué doctrina se elaboró, qué se resolvió frente a un problema" (Gordillo, 2000, p.131).

Socorro y Cruceta (2007), dejan ver que la lectura minuciosa de los géneros jurídicos, específicamente en lo que se refiere a sentencias, demanda el uso de competencias críticas para:

1. Identificar los motivos, aspectos en los que confluyen las pretensiones y los medios aportados por las partes y, de los cuales dependió en cierto modo la resolución aplicada por el juez.

2. Determinar la jurisdicción, elemento que desde el punto de vista técnico y del aprendizaje, le permite al lector conocer la forma como se jerarquiza y ejercen control los órganos jurisdiccionales del Estado.

3. Revisar los argumentos y las conclusiones favorece que el lector logre valorar la correspondencia, pertinencia y precisión que asumió el juez en procura de administrar justicia.

4. Lo anterior debe guiar al lector a la comprensión de elementos explicativos que subyacen a toda sentencia, y de los que depende su justificación. Estos suelen unir las pruebas que apoyan la decisión y la respectiva fundamentación jurídica que sustancia la posición del juez.

Otras perspectivas sobre la lectura crítica proponen la necesidad de valorar de manera profunda el contenido de los documentos jurídicos, en el caso de una sentencia se pide la identificación de las secuencias discursivas que esta contiene, es decir, la revisión de 
los apartados en los que se ofrece explicaciones de los hechos, los cuales en ocasiones se encuentran claramente expuestos en los considerandos y las tramas que definen aspectos medulares (Guerra, 2006; Morales, 2020); a lo dicho se agrega, la necesidad de asumir para el análisis la identificación de dos variables "prestar atención a lo que el fallo resuelve frente a un problema determinado, y b) prestar primordial atención a los argumentos que el fallo despliega" (Gordillo, 2000, p. 133). A manera de complemento, las propuestas derivadas de la lectura en ciencias sociales dejan ver la necesidad de ir sobre el documento para identificar el objetivo 0 propósito de la querella, los aspectos o exigencias que hacen parte del petitorio y, lo que en la sentencia se resuelve frente a una problemática social.

En función de lo anterior, se proponen algunas estrategias a partir de las cuales el docente y el lector novato pueden lograr que su acercamiento al conocimiento jurídico adquiera sentido, significado y aplicabilidad en la praxis. Estas son:

1. El lector acucioso debe de identificar aquellos apartados, ideas y planteamientos en los que el legislador no logró motivar con suficiente claridad sus pretensiones. Para ello es necesario que apoye su comprensión con la lectura de textos complementarios que le ayuden a completar aquello en lo que considera la existencia de vacíos (Morales, 2020; Moretó, 2014).

2. Identificar los argumentos que componen la estructura retórica le permite al lector además de mayor rigurosidad académica, entender cómo se organizan las definiciones, las relaciones causa efecto, los argumentos analógicos y la ejemplificación, recursos que el legislador utiliza para sustanciar y motivar las sentencias y decisiones jurisprudenciales.

3. La lectura crítica de textos jurídicos exige el uso de habilidades cognitivas para determinar cuándo se está frente a secuencias explicativas, descriptivas 0 argumentativas a las que el legislador o jurisconsulto apela para demostrar la validez de sus planteamientos (Arenas, 2009).

4. El uso de textos complementarios permitirá identificar las actuaciones desarrolladas sin el sustento informativo suficiente así como otros errores que pudieran tornar tendenciosas 0 subjetivas las decisiones tomadas así como guiar a procedimientos errados o ilógicos. Esta estrategia le aportará al lector, los elementos necesarios para reconstruir y completar las ideas faltantes y, de este modo, contar con un panorama general de la situación en conflicto (Guerra, 2006; Morales, 2017).

5. Es necesario según Moretó (2014), que el lector defina entre otras cosas "el propósito de su lectura, se cuestione, busque la información relevante, haga inferencias, sintetice y monitoree la construcción del significado" (p.100).

6 . El rol del docente en las prácticas de lectura guiada debe enfocarse en la activación de procesos mentales que le permitan al lector "problematizar, elaborar preguntas, volver sobre el texto, realizar predicciones y construir hipótesis sobre el significado del texto (Moretó, 2014, p.114).

7. Como estrategia retórica, es necesario que el lector en su rol activo logre "relacionar el caso tratado en la sentencia con los conocimientos que maneja de casos similares, es decir, otras resoluciones judiciales y con aportes doctrinales que le cooperen con la comprensión de la solución que se le da al caso" (Moretó, 2014, p. 115).

8. En el caso de documentos jurídicos, caso de las sentencias judiciales, es importante que el lector identifique los hechos, las pretensiones expuestas por el querellante y los fundamentos 
Lectura en derecho: sugerencias para guiar al estudiante en su proceso formativo y en la apropiación del conocimiento

que el juez asumió de las fuentes doctrinales para resolver el caso en controversia (Weston, 2006).

Otras estrategias para el análisis de textos académicos de la disciplina jurídica, encuentra sus referentes en Anchondo (2016), Borja (2007) y Knorr (2010) y se enfocan en los siguientes aspectos:

1. Se hace necesario identificar el tipo de documento que se leerá. Esto implica ubicarlo dentro de los géneros de manejo frecuente en el campo del derecho: leyes, tratados, jurisprudencias, doctrinas y contratos.

2. Es fundamental que el lector sitúe el documento en el área o las áreas a las que pertenece la controversia tratada, es decir, si responde a derecho privado, público, civil, mercantil, penal, laboral 0 administrativo.

3. Buscar otras fuentes doctrinales y jurisprudenciales ayudan con la elaboración de conclusiones completas; esta estrategia favorece que se profundice y establezcan analogías como parte de las operaciones mentales que dan lugar a la construcción amplia de ideas.

4. Determinar el ámbito de aplicación al que va dirigido el procedimiento judicial, permite delimitar el alcance que tiene la decisión: regional, nacional e internacional.

5. En todo documento por existir una relación jurídica derivada de un acuerdo entre las partes. Esto demanda que el lector defina con precisión figuras como el sujeto pasivo y el sujeto activo, así como los vínculos y condiciones que los une en el nexo establecido (relación jurídica).

6. La lectura de una ley o norma usualmente demanda de la revisión literal de su intensión; sin embargo, es importante que el lector determine, por un lado, el régimen sancionatorio y, por el otro, tipifique las infracciones. Esta operación desde el punto de vista instrumental le permitirá contextualizar aquellas conductas o comportamientos susceptibles de sanción.

7. Una actitud más acuciosa pudiera llevar al lector a: revisar el contexto sociopolítico en el que la norma es discutida, aprobada y promulgada; determinar si la misma responde con efectividad a los ideales de justicia, igualdad y bien común; y, si las sanciones son las adecuadas para proteger al sujeto para el que fue destinada, reprimiendo con ello las conductas que pudieran atentar contra el débil jurídico.

Con respecto a lo anterior, la lectura crítica propone algunas ideas sobre el análisis y la interpretación de normas jurídicas, que pudieran ser aplicadas de manera global a la lectura crítica de leyes, reglamentos, estatutos y tratados, estas son:

1. La interpretación de una norma requiere no solo de la búsqueda de sentido y su alcance, sino deducir la finalidad que persigue, actuaciones que regula y posibles sanciones que se derivan en caso de cometer alguna infracción de las contenidas en ella.

2. Entender una norma exige la revisión de los factores históricos, sociales, culturales, políticos y económicos.

Lo dicho se indica que, la lectura de leyes demanda el uso criterios interpretativos para descubrir e identificar las intenciones del legislador así como los principios, contenidos implícitos y explícitos que definen su existencia como norma social. Estos criterios refieren a: posibles soluciones propuestas en la norma, principios jurídicos invocados, en función de los cuales se motiva la regulación de situaciones específicas o generales que pudieran referir a otras fuentes en las que, el lector haciendo uso del criterio sistemático de análisis normativo debe acudir a normas del sistema jurídico con competencia en la regulación directa o 
indirectamente de la misma institución y, finalmente, apelar al elemento histórico posibilita la interpretación objetiva de la norma al determinar cuál fue la voluntad del legislador y el significado real atribuido en el momento que fue formulada.

Socorro y Cruceta (2007), proponen que la lectura de doctrina debe involucrar la valoración profunda y acuciosa de las afirmaciones del autor, condición que exige varias operaciones, entre las que se mencionan "reconocer los razonamientos cuando aparecen; identificar sus premisas y sus conclusiones; reconstruirlos explicitando los enunciados tácitos" (p.115). En el caso de textos argumentativos, por su complejidad, deben ocupar al lector en la revisión interpretativa, habilidad que le permitirá integrar información y la mayor parte de los contenidos para luego reconstruir el conocimiento mediante generalizaciones que aporten de modo significativo al campo disciplinar.

León (2008), afirma que un buen lector debe ser capaz identificar en un documento jurídico (demanda) elementos específicos como "el problema y sus componentes, las alegaciones de las partes, los elementos fácticos y normativos así como las motivaciones que los involucrados se exigen para resolver el objeto de la querella. Interpretando a Gimeno (2000) de una lectura crítica y activa depende la resolución oportuna de un conflicto, por ende, esta debe estar orientada a la búsqueda de los hechos o supuestos que hacen parte de la controversia y, si estos encajan en el campo de aplicación de una u otra norma jurídica a la que el juez o abogado pudieran acudir para ofrecer soluciones apegadas a derecho.

En el caso de otros géneros jurídicos como las sentencias, es importante que el lector se aproxime a la identificación de los siguientes aspectos: revisión de los antecedentes 0 de la historia procesal del caso; situación objeto del conflicto; instancias judiciales en las que se ventiló la querella, actuaciones y respuestas emanadas; tramas o nudos controversiales de los que se desprendieron las soluciones; razonamientos, planteamientos y elementos doctrinales utilizados por el juez para sustanciar y argumentar su decisión (Atienza, 2005; Morales, 2020) Vázquez, 2009).

Otros documentos como los contratos, ameritan según Gordillo (2000), otro modo de leer enfocado en identificar los hechos que llevaron a celebrar el acuerdo, las conductas reguladas, los acuerdos y las posibles situaciones que pudieran llevar a la disolución del contrato así como a la atribución de responsabilidad civil 0 administrativa a las partes que integraron las relación jurídica. Knorr (2010), aporta algunas sugerencias que el lector debe asumir como parte de su aprendizaje estratégico, estas son: leer textos jurídicos exige el uso de habilidades para identificar secuencias discursivas particulares como la narración de hechos, la explicación de situaciones y la descripción; formular hipótesis constituye una operación importante que requiere del manejo de ciertas ideas y conocimientos previos que favorecen el acercamiento a los elementos resolutorios en una sentencia o jurisprudencia; identificar el contexto y la problemática que configura la controversia facilita la construcción panorámica de la trama expuesta en el documento jurídico.

Aportes derivados de la lectura crítica y la argumentación en Derecho proponen que su objetivo debe enfocarse en la construcción y reformulación de argumentos con el propósito de organizar enunciados e integrar premisas para que pudieran favorecer la comprensión de las temáticas propuestas en los textos científicos. Esto en palabras de Socorro y Cruceta (2007) exige asumir una posición reflexiva para "definir elementos conexos, aspectos relevantes y 
Lectura en derecho: sugerencias para guiar al estudiante en su proceso formativo y en la apropiación del conocimiento

afirmaciones sobre las que el autor sustenta sus conclusiones o apoya la postura que intenta defender" (p.114).

Anchondo (2016), deja entrever que la lectura jurídica procura varios propósitos entre los que destacan "lograr que el significado que se asigne a una ley o contrato sea el más razonable y convincente y evitar que sea simplemente el resultado de la particular apreciación, de una visión limitada de la posible solución" (p.34). Estos cometidos desde la lectura crítica exigen desentrañar el verdadero significado de una norma, determinando de este modo las intencionalidades reales, las posibles vías a las que se pudiera apelar para resolver potenciales querellas judiciales y los niveles de efectividad en cuento alcance se refiere.

Otras perspectivas relacionadas con la lectura y el análisis crítico de textos académicos, proponen que el acercamiento significativo al conocimiento científico requiere de actividades cognitivas como: identificar el argumento principal al que el autor apela para fijar su posición, este generalmente se encuentra soportado en otras ideas que lo sustancian; revisar la consistencia de las evidencias, las cuales puedes representar hallazgos de terceros que le aportan mayor credibilidad y validez a su tesis; y, como parte de las habilidades que deben activarse en el lector en su proceder crítico se mencionan: hacer uso de las inferencias (lugar, tiempo, entre otras) comparaciones, buscar explicaciones y valorar la veracidad de los razonamientos.

Involucrar a los estudiantes en este cúmulo de operaciones mentales, favorece que en su desempeño académico y profesional logre según expone Álvarez (2008), el "interpretar y aplicar el derecho, describir y narran hechos, formular peticiones, demostrar sus razonamientos mediante la argumentación, emiten juicios, expida órdenes, elabore las condiciones que conciertan los contratos, exprese y conteste agravios y, produzca y responda alegatos" (p.138). Adicionalmente la autora agrega que, la apropiación del conocimiento como resultado de la lectura crítica, persigue entre otros propósitos "lograr la adhesión del auditorio, a refutar las tesis adversas, a insertar el discurso en el sistema de valores imperantes 0 a producir una ruptura con éste" (p.139).

Para Cardinale (2007), la lectura y el aprendizaje de contenidos en la universidad implica "apropiarse de los sistemas conceptuales y metodológicos, así como de las prácticas discursivas de las que depende el cambio de identidad como pensadores y analizadores de textos, capaces de identificar las polémicas que subyacen en su campo del saber" (p.3). Por su parte Weston (2007), propone que las bondades de la lectura se encuentran indefectiblemente vinculadas con el pensamiento crítico, por lo que el desarrollo de ejercicios en los que se objete, critique y valore con rigurosidad las ideas, representa un medio para que el estudiante con criterio fundamente "la revisión de sus razonamientos, cuestione creencias, y someta a prueba y defienda sus propios puntos de vista" (p.10).

Se trata entonces de involucrar al lector en situaciones de aprendizaje a través de las cuales se vea en la necesidad de aprender a pensar de manera autónoma, cuestión que implica según Weston (2008), el "formar sus propias razones y ofrecer alternativas de solución que aporten respuesta la situación en conflicto" (p.10). Esto implica que el lector haga uso de la indagación como una actividad inherente a su formación, y de la que depende soportar los argumentos que utilizará para explicar y defender sus propios alegatos.

Para Ulloa, Crispín y Béjar (2014), la lectura de textos académicos en cualquier disciplina debe orientar al estudiante al uso de habilidades cognitivas como el análisis y la 
síntesis, las cuales "llevarán a comprender contenidos y expresar sus propias ideas y puntos de vista argumentando correctamente; para ello se hace inminente el desarrollo de lecturas atentas y reflexivas en las que se dialogue con los autores haciéndoles preguntas pertinentes" (p.2). Esta manera de interactuar con el conocimiento posiciona al lector como un agente activo que, valiéndose de su sentido crítico es capaz de identificar los planteamientos fundamentales a los que el autor le dedica especial atención así como las discusiones que este genera para dejar por sentada su posición frente a la temática abordada.

Por su parte Atienza (2005), deja ver en su perspectiva de la argumentación jurídica una serie de ideas sobre la lectura, proceso al que entienden como la forma de justificar y darle validez a los acontecimientos que se dan en el mundo social. De allí la afirmación en la que indica que "leer favorece la identificación de las premisas y conclusiones a las que llega un autor, tarea que demanda importantes dosis de análisis que le permitan al lector confrontar lo dicho con lo que sucede en la realidad" (p.5). Agrega el autor que, leer de modo crítico una norma o ley debe involucrar actividades como el contexto social, las condiciones económicas, los móviles psicológicos y las circunstancias ideológicas en la que fue elaborada y promulgada; de esto dependerá que los administradores de justicia logren dictar resoluciones sustentadas de manera aceptable y correcta.

En apoyo a esta postura, Atienza (2005), deja ver que la lectura acuciosa constituye la puerta de entrada a prácticas comunicativas eficaces, al debate apropiado y la formulación de razonamientos desde los cuales cuestionar y "defender un punto de vista con el que se pretende modificar la conducta del otro, mediante ideas más elaboradas que superen otras alternativas y cuyo poder de adhesión comprometa a la audiencia" (p.5). Lograr este cometido exige que el lector "construya o reconstruya un juicio normativo que será la premisa a partir de la cual dará oportuna solución al caso en cuestión" (Vigo, 2006, p.276). Ello demanda el ser capaz de ir sobre el pensamiento del legislador en una actitud interpretativa que busque el verdadero significado de la norma, y las posibles respuestas jurídicas que frente al caso el sistema y su ordenamiento invocan para resolver su funciones como: el orden, la justicia, el bien común y la equidad.

Gordillo (2000), manifiesta que convencer a una audiencia obliga al lector a recurrir a elementos doctrinales que asumidos como argumentos de autoridad, le otorgan mayor credibilidad a su posición; esto implica desde el proceder ético del abogado que se forma, la disposición para asumir con "honestidad, responsabilidad, seriedad y de manera racional frente al problema que procura resolver" (p.123). De este modo, por reposar en el abogado un elevado compromiso social e intelectual, se le demanda el uso de la valoración justa y ecuánime como medio para identificar desde diversos ángulos posibles alternativas que le cooperen con la demostración de su tesis, de la cual espera que los órganos decisores se sumen a sus planteamientos.

Lo anterior refiere entonces, al compromiso social y ético que debe dirigir al jurista, de quien se espera no solo la aplicación de argumentos teórico-conceptuales y normativos, sino el uso de competencias críticas que faciliten la comprensión profunda y le indiquen cómo "escudriñar de manera profunda en las circunstancias que motivaron el hecho y de las cuales depende significativamente que el administrador de justicia haya elaborado determinados argumentos o razones para sustanciar la resolución del caso" (Gordillo, 2000, p.135). Ello indica, hacer uso la inferencia y el pensamiento deductivo como operaciones mentales 
Lectura en derecho: sugerencias para guiar al estudiante en su proceso formativo y en la apropiación del conocimiento

relacionadas con el análisis y la construcción de lo sucedido, para lo que necesita atender a elementos como el estudio del lugar, el tiempo y las condiciones que participaron en el hecho jurídico.

De allí que sea imprescindible capacitar al lector para que desarrolle de manera efectiva actividades como: la reconstrucción de hechos y situaciones, para lo cual se hace necesario el uso de la inferencia, el análisis y la interpretación, habilidades que por sus implicaciones permiten la elaboración de alegatos y argumentos consistentes de los que depende que en un proceso judicial se sentencie 0 absuelva a las partes que hacen parte del conflicto. Esto en la praxis jurídica significa lograr que el sujeto decisor se adhiera a la posición de quien defiende o refuta, pues en ambas partes se encuentra el compromiso de dejar sin efecto los recursos propuestos por su contraparte, actuación que depende de la valoración crítica de las tesis adversas que pudieran ser contraproducentes al momento de resolver una querella judicial.

De los planteamientos de Suarez y Conde (2009), se infiere que, la lectura académica exige el manejo de competencias críticas que cooperen con determinar la validez y racionalidad de toda decisión jurídica, la cual pudiera presentarse de modo silogístico, es decir, conformado por premisa mayor y menor, cuyos contenidos se organizan para sustanciar la postura que se intenta defender y, que a su vez, vienen a constituirse en argumentos que buscan validar un proceso resolutivo.

En suma, leer en ciencias jurídicas constituye no solo un proceso disciplinado y acucioso, sino un compromiso con la formación académica de los que en un futuro cercano se encargarán de la administración de justicia. Por ende se espera que lector del Derecho disponga de habilidades críticas y analíticas que agudicen su pensamiento, instrumento que por su potencial constituye el medio para construir soluciones y escoger alternativas que, a su vez posibiliten procesos resolutorios coherentes y permeados por la convicción de quien por su experticia es capaz de propiciar la toma de decisiones lógicas, viables y apegadas a Derecho.

\section{Experiencia de enseñanza de la lectura de textos jurídicos}

La lectura de textos jurídicos con nuevos ingresantes a la universidad representa un desafío para el docente, dado que por lo general esta población ha tenido escasas experiencias significativas, capaces de potenciar su pensamiento y el desarrollo de competencias críticas, en función de las cuales operar sobre el conocimiento que circula en las disciplinas. En tal sentido, este apartado sintetiza las principales reflexiones generadas en torno a seis (06) sesiones de lectura académica realizadas con estudiantes del ciclo introductorio de Derecho de la Universidad de Los Andes. Los materiales seleccionados fueron textos del género periodístico, con temáticas controversiales y propias del contexto nacional e internacional, de las cuales se precisan: la eutanasia, el aborto, los derechos humanos emergentes, legalización de las drogas, democracia, las libertades individuales y colectivas.

Esta experiencia de lectura permitió proponer las siguientes reflexiones cuyo objetivo es mejorar la praxis docente. En primer lugar, la integración y participación activa de los estudiantes en las discusiones correspondientes a cada sesión, estuvieron motivadas por la vigencia y pertinencia de las temáticas seleccionadas; los participantes indicaron su acuerdo y desacuerdo, su posicionamiento personal y las miradas disciplinares existentes en torno a los tópicos controversiales a través de opiniones personales. Esto indica, que la interacción 
significativa con el conocimiento parte, entre otras cosas, de la calidad, actualidad y adecuación de los materiales ofrecidos por el docente a quienes intentan afiliarse a una nueva cultura académica, con prácticas y convenciones sociales particulares (Cardinale, 2007).

En segundo lugar, el uso de la lectura guiada debe entenderse como un proceso de acompañamiento en el que tanto el docente como el lector novato revisan con detenimiento cada apartado del texto, sus ideas, argumentos, contraargumentos, miradas disciplinares, entre otros. Durante la experiencia de lectura, se logró la identificación de aspectos importantes como: ideas subyacentes, planteamientos explícitos, posturas ideológicas, argumentos de autoridad, ejemplificación, causalidad y analogía, así como la tesis y sub-tesis del autor. Integrar la lectura guiada en cada sesión contribuyó con la comprensión y la adopción del diálogo profundo y significativo con el saber, el mejoramiento de las prácticas de producción de conocimiento propio de la praxis jurídica, así como la integración de los recursos retóricos a los que el jurista apela para sustanciar cualquier documento, sentencia 0 jurisprudencia.

En tercer lugar, la promoción del pensamiento crítico dependió en modo significativo del carácter controversial de las temáticas propuestas y del posicionamiento de los lectores. Para gran parte de los estudiantes la crítica se asoció con la manifestación de acuerdo o desacuerdo con las ideas del autor, por lo que emitieron juicios orales y manifestaron su postura en sus producciones escritas, construyeron valoraciones personales, objetaron planteamientos ideológicos y supuestos, a los que consideraban tendenciosos o falaces. En cuarto lugar y como habilidad cognitiva indispensable para quien se forma y ejerce el Derecho, se motivó el manejo de la argumentación como resultado del razonamiento minucioso y de la interpretación profunda de las ideas propuestas (Anchondo, 2016); parte de las evidencias indican que los estudiantes junto a su posición sobre la temática, ofrecieron razones y premisas, así como posibles alternativas o soluciones no estimadas por el autor en torno a la problemática social tratada.

En quinto lugar, al inicio del curso se evidenció el manejo deficitario de estrategias de lectura; sin embargo, a largo de cada sesión los estudiantes activaron sus conocimientos previos, definieron propósitos de lectura, se apropiaron de la predicción, dialogaron con el autor mediante preguntas, subrayaron o destacaron en el texto, identificaron elementos retóricos, elaboraron hipótesis y las sometieron a comprobación, como estrategias que permitieron la construcción de opiniones propias (Moretó, 2014). En sexto lugar, se logró el manejo de competencias críticas como determinantes de la autonomía asociada con el rendimiento académico. Los estudiantes mostraron su competencia para problematizar, leer en varias direcciones identificando premisas disciplinares, contextualizaron los contenidos mediante la integración de los conocimientos previos con lo aportado por los textos. A esto se suma el desarrollo de habilidades cognitivas importantes como: la síntesis, el análisis, la argumentación, la explicación y la interpretación, como operaciones de las que depende el ejercicio profesional competitivo del administrador de justicia.

En séptimo lugar, es necesario indicar que la creación de un ambiente constructivo motivado por la flexibilidad del docente con respecto a las intervenciones de los estudiantes, redundó en la consolidación del aprendizaje significativo y en el desarrollo de las competencias, aptitudes, habilidades y destrezas para interactuar con el conocimiento científico. Esto sugiere para quien enseña el proceso de lectura, la motivación en el aula de 
Lectura en derecho: sugerencias para guiar al estudiante en su proceso formativo y en la apropiación del conocimiento

clase un ambiente plural y democrático que privilegie la libertad para participar, opinar y emitir juicios valorativos, acuerdos y desacuerdos, frente a los cuales la actuación del docente debe girar en torno a reconducir la discusión y el diálogo hacia propósitos académicos.

Lo anterior debe entenderse como el respeto a las ideas, creencias y pensamientos de cada estudiante, como requerimiento del cual depende su integración a las convenciones académicas establecidas por cada disciplina, así como la adquisición de estrategias para interpretar textos académicos, doctrinales y normativos para operar dentro del mundo jurídico con pertinencia. En tal sentido, es perentoria del docente como actor capaz de impulsar la apropiación de conceptos claves, que unidos a los conocimientos previos permitan progresivamente ingresar a contenidos complejos a lo largo de su carrera profesional.

En suma, esta experiencia de lectura académica permitió que los estudiantes del ciclo introductorio de Derecho lograran ir más allá de lo expuesto en los textos, hasta la generación de nuevos conocimientos válidos y pertinentes, como condiciones necesarias para sustanciar la toma de decisiones responsables en torno a la resolución de problemas sociales. Este acercamiento a la cultura jurídica impulsó el manejo de estrategias básicas asociadas con: el establecimiento de relaciones, el uso de la comparación, del análisis crítico y de la apropiación de vocabulario que a futuro deberá utilizar en su ejercicio profesional.

\section{Consideraciones finales}

La formación jurídica en el presente más que en cualquier momento enfrenta retos importantes, determinados entre otras razones por: la complejidad de la cultura académica jurídica, la abundante información y por el carácter dinámico de la vida social, condiciones que exigen del lector que se forma en este campo del saber, no solo su actitud crítica y analítica; sino su experticia para acercarse selectivamente a los documentos que mayormente se consultan y revisan para comprender el hecho socio-jurídico y las relaciones en las que el Derecho además de regular, permite definir las acciones que, tanto administradores de justicia como particulares deben conocer para dirimir oportunamente los conflictos que emergen de la vida social.

De este modo, leer en ciencias jurídicas demanda la participación activa del sujeto y, en especial, el desarrollo de habilidades y competencias críticas a través de las cuales dispongan el pensamiento para dilucidar las decisiones oportunas y convenientes, que le permitan ejercer de manera eficiente sus funciones como administradores de justicia, decisores (jueces) o parte en una situación jurídica, en la que se espera sea capaz de "formular hipótesis, generar soluciones, hacer uso de la comparación y el análisis para definir causas y consecuencias, describir y narrar hechos y procesos, que por representar información valiosa le aportan las referencias para explicar con experticia un fenómeno socio-jurídico" (Fernández y Ramírez, 2011, p.24).

Con relación a la experiencia de lectura académica, es preciso hacer referencia al compromiso del docente como agente promotor de la afiliación, integración y participación del estudiante en la cultura académica de cualquier disciplina. Las interacciones con el saber jurídico permitieron no solo el desarrollo de competencias críticas y habilidades cognitivas de orden superior, sino operar crítica, analítica y reflexivamente sobre supuestos teóricos, posturas ideológicas y premisas reconocidas científicamente, pero que al ser sometidas al 
pensamiento crítico resultaron falaces, carentes de veracidad y potencialmente rebatibles; este proceder supone manifestación de rasgos propios de la autonomía, como actitud asociada con la capacidad para interactuar, dialogar, razonar, realizar inferencias y ofrecer soluciones a problemas reales.

En síntesis, las prácticas de lectura en la universidad y en especial en áreas relevantes para la sociedad como el Derecho, ameritan de un elevado compromiso de quien aprende, debido a que este nivel se exige el uso de habilidades el pensamiento para criticar, analizar y reflexionar sobre fenómenos complejos en torno a los cuales giran posiciones contrapuestas, diversas y controversiales que deben revisarse de manera profunda, con el objeto de disipar dudas, detectar interpretaciones tendenciosas y, como resultado de ello proceder atendiendo al verdadero espíritu de las leyes, normas y procedimientos vigentes para el conflicto en cuestión.

\section{Referencias bibliográficas}

Alvarado, Patricia. (2014). El desarrollo del pensamiento crítico: una necesidad en la formación de los estudiantes universitarios. Revista Didáctica, 64. México. (Pp.10-17). http://revistas.ibero.mx/didac/articulo detalle.php?id_volumen=18\&id_articulo=216

Anchondo, Victor. (2016). Métodos de interpretación jurídica. Universidad Autónoma de Chihuahua. México.

Arenas, Stella. (2009). Criterios generales para el desarrollo de las habilidades para la comprensión y producción de textos escritos académicos. Universidad Nacional. Lectura y vida. Revista Latinoamericana de Lectura. Argentina. (Pp. 1-10).

Atienza, Manuel. (2005). Las razones del derecho. Teorías de la argumentación jurídica. Universidad Nacional Autónoma de México. México.

Bhatia, Vijay Kumar. (1997). Applied genre analysis and ESP. En T. Miller (ed.), Functional approaches to written text: Classroom applications. Chinese University of Hong Kong. China.

Borja, Anabel. (2007). Los géneros jurídicos. Alcaraz (ed.). Las lenguas profesionales y académicas. Editorial Ariel. España.

Cardinale, Lidia. (2007). Lectura y escritura en la Universidad. Aportes desde la práctica pedagógica. Revista Pilquen-sección pedagógica. Año VII, N 3, 2006/2007. Argentina. (Pp. 1-5). https://dialnet.unirioja.es/descarga/articulo/3055578.pdf

Dolz, Joaquín y Pasquier, Auguste. (1997). Argumentar para convencer. Recursos didácticos. Departamento de Educación y Cultura. Edita Gobierno de Navarra. España.

Fernández, Hilda y Ramírez, Rogerio. (2011). Leer para investigar. Revista La Colmena, octubre-diciembre.

México.

(Pp.23-29).

http://web.uaemex.mx/plin/colmena/Colmena_72/Aguijon/Leer_para_investigar.pdf

Gimeno, María. (2000). Teoría y doctrina de la interpretación jurídica propuesta por De Riccardo Gauistini. Universidad de León. Doxa, n²3. España. (Pp. 689-707). https://rua.ua.es/dspace/bitstream/10045/10283/1/doxa23_27.pdf

Gordillo, Agustín. (2000). Introducción al Derecho. Fundación de Derecho Administrativo. Argentina.

Guerra, Juan. (2006). La lectura crítica de textos jurídicos. Revista Universitas, $n^{\circ} 4$. España. (Pp. 69-81). http://universitas.idhbc.es/n04/04-04.pdf 
Lectura en derecho: sugerencias para guiar al estudiante en su proceso formativo y en la apropiación del conocimiento

Hawez, Gustavo. (2003). Pensamiento crítico en la formación universitaria. Universidad de Talca. Revista del Instituto de Investigación y Desarrollo Educacional. Disponible http://www.pregrado.utalca.cl/docs/pdf/documentos_interes/Pensamiento $\% 20$ Critico $\% 2$ 0en\%20la\%20Formacion\%20Universitaria.pdf

Knorr, Patricia. (2010). Estrategias para el abordaje de textos. Material didáctico. Universidad Nacional de General Sarmiento. Argentina.

León, Ricardo. (2008). Manual de redacción de resoluciones judiciales. Academia de la Magistratura. Perú.

Morales, Jesús. (2017). Pensamiento crítico y lectura en ciencias sociales. Revista

Electrónica Calidad en la Educación Superior. Vol. 8(2). Costa Rica (Pp.265-282). https://doi.org/10.22458/caes.v8i2.1943

Morales, Jesús. (2018). Aportes de Paulo Freire a la Investigación y a la Lectura Crítica.

Revista Internacional de Educación para la Justicia Social, 7(2). España (Pp.175-192). https://doi.org/10.15366/riejs2018.7.2.010

Morales, Jesús. (2020). Leer en la universidad: algunas reflexiones para mejorar su práctica.

Revista la Pasión del Saber, $N^{\circ}$ 17, enero-julio 2020. Venezuela. (Pp.1-25). http://201.249.74.131/pasion/index.php/using-

joomla/extensions/templates/protostar/home-page-protostar/98-edicion-17/262-leer-enla-universidad-algunas-reflexiones-para-mejorar-su-practica

Moretó, Martín. (2014). Estrategias de lectura de textos jurídicos: análisis de protocolos verbales en expertos y novatos. Revista Científica de Vol. XVIII № 1 -Primavera. Argentina.

(Pp.97-120).

http://dspace.uces.edu.ar:8180/xmlui/bitstream/handle/123456789/2615/Estrategias_Mor eto.pdf?sequence $=1$

Pascual, Rosana. (1997). La argumentación: Nociones generales. EN: Romero, Daniel, comp. Propaganda política: Discurso y argumentación. Buenos Aires: Los Libros del Riel. Argentina. (Pp. 41-49). En Memoria Académica. Disponible http://www.memoria.fahce.unlp.edu.ar/libros/pm.430/pm.430.pdf

Pettoruti, Carlos. (2005) ¿Qué es el Derecho? Curso de adaptación universitaria. Universidad de la Plata. Argentina.

Socorro, Juan. y Cruceta, José. (2007). Argumentación jurídica. Escuela Nacional de la Judicatura. República Dominicana.

Suarez, Miguel.,y Conde, Napoleón. (2009). Argumentación jurídica. Universidad Autónoma de México. México.

Ulloa, José., Crispín, María y Béjar, Ofelia. (2014). La lectura y la escritura ¿se deben aprender en la universidad? Programa de Formación de Académicos. Nº10. México. (Pp. 17). $\quad$ http://crea.um.edu.mx/wp-content/uploads/2017/03/Ulloa-Herrero-et-alLa lectura la-escritura.pdf.

Vázquez, Omar. (2009). De lo que la teoría de la argumentación jurídica puede hacer por la práctica de la argumentación jurídica. Revista Telemática de Filosofía del Derecho, no 12. México. (Pp.99-134).

Vigo, Rodolfo. (2006). Ética judicial e interpretación jurídica. DOXA, Cuadernos de Filosofía del Derecho, 29. España. (Pp. 273-294). https://doi.org/10.14198/DOXA2006.29.14

Weston, Anthony. (2006). Las claves de la argumentación. Editorial Ariel, S. A. España. 